\title{
Determination of the Modal Abundance of Nano-Scale Amorphous Phases Using Selected Area Electron Diffraction Mapping.
}

\author{
Bradley De Gregorio ${ }^{1}$, Rhonda Stroud ${ }^{1}$, Neyda Abreu ${ }^{2}$, and Kieren Howard ${ }^{3}$ \\ 1. Naval Research Laboratory, Materials Science and Technology Division, Washington, DC USA. \\ 2. Pennsylvania State University DuBois, Department of Earth Sciences, DuBois, PA USA. \\ 3. American Museum of Natural History, New York, NY USA.
}

Measurement of modal abundances in a nanophase composite material can be accomplished using various analytical methods (WDS in an electron microprobe, STEM-EDS, or EBSD/TKD in a SEM). However, these analytical techniques can fail when some of the nanophases of interest are amorphous or glassy. WDS and EDS measure the composition of the target material, and a structurally-amorphous silicate material may have the same elemental composition as a crystalline silicate mineral. For EBSD and TKD, amorphous materials will show no Kikuchi lines in the measured diffraction pattern, and may therefore, be confused with low quality patterns from other crystalline materials or void space. One alternative for differentiating compositionally-similar amorphous and crystalline materials is selected area electron diffraction (SAED) in a conventional TEM. SAED patterns from crystalline materials will contain diffraction spots (or sharp rings if they are 2-10 $\mathrm{nm}$ in size), while those from amorphous materials will have only broad rings. However, to calculate modal abundances of nanophases in an amorphous/crystalline composite material using SAED, each grain must be visited, tilted to a zone axis crystal orientation (if crystalline), and then imaged to measure the grain size. This approach is prohibitively time consuming for areas greater than $1 \mu^{2}$.

We have developed an automated approach to overcome the limitations of using SAED to measure the modal abundance of amorphous silicate over a larger area in a nanophase composite. This method will be first applied to a thin lamella of fine-grained matrix extracted from the meteorite ALH77307 using focused ion beam methods. The matrix of ALH77307 contains abundant amorphous silicate material [1]. During the evolution of asteroids, the parent planetary source of meteorites, amorphous silicates are known to be rapidly destroyed by annealing or hydration. The presence of amorphous silicate in meteorites is evidence that they preserve a record of the earliest processes and components active in the ancient Solar System, and their modal abundance can be used as a measure of "primitiveness", or the absence of later alteration of the initial components [2].

The SAED patterns from the ALH77307 FIB lamella were collected with a $200 \mathrm{keV}$ field-emission JEOL 2200FS at the Naval Research Laboratory. The microscope is equipped with an in-column "omega" energy filter, so a $5 \mathrm{eV}$ filter slit was introduced, centered on the zero-loss peak, to significantly reduce diffuse scattering. Diffraction patterns were produced using a $200 \mathrm{~nm}$ SAED aperture (Figure 1), selected to optimize tradeoffs in spatial resolution, stage reproducibility, and dataset size. Using this aperture size, more than one phase was often present in the SAED patterns, since the grain size of the meteorite matrix ranges from 5-500 nm. A custom script was created for Gatan DigitalMicrograph $\odot$ to translate the sample stage across the FIB lamella at $200 \mathrm{~nm}$ steps and acquire 2,162 $512 \times 512$ pixel SAED images with a Gatan Ultrascan 1000 digital camera, covering a total area of $5.5 \times 5 \mu \mathrm{m}$. The stage position reproducibility was observed to be $\pm 100 \mathrm{~nm}$, which limited the spatial resolution of this technique, but further improvements can likely be achieved by modifying the script to account for stage drift. The SAED images were processed offline with custom Python code. 
Radial intensity profiles (RIPs) of SAED patterns from crystalline grains contain several sharp peaks, while those from purely amorphous silicate are smooth and show increased intensity at low radii (Figure 1). RIPs from mixed amorphous/crystalline material have intermediate characteristics, with increased intensity at low radii and small sharp peaks. Diffraction peaks were identified directly on the SAED patterns using a $24 \times 24$ pixel local maximum image filter, and the smooth amorphous ring patterns were subtracted using a similar local minimum image filter. Initially, the mean intensity of the resulting diffraction peak image was used as a simple proxy for the crystalline/amorphous abundance in each SAED pattern (Figure 2), but more sophisticated calculations may also be used. By inspection, a threshold value for $100 \%$ amorphous silicate was determined and applied to the crystallinity map to produce a map of pure amorphous silicate. Crystalline grains larger than 5-10 nm can be mapped by selecting SAED patterns with low average image intensity near the center.

\section{References:}

[1] AJ Brearley, Geochimica et Cosmochimica Acta 57 (1993), p. 1521.

[2] NM Abreu and AJ Brearley, Geochimica et Cosmochimica Acta 74 (2010), p. 1146.

[3] The authors acknowledge funding from the NASA Cosmochemistry Program, grant NNX14AG27G.
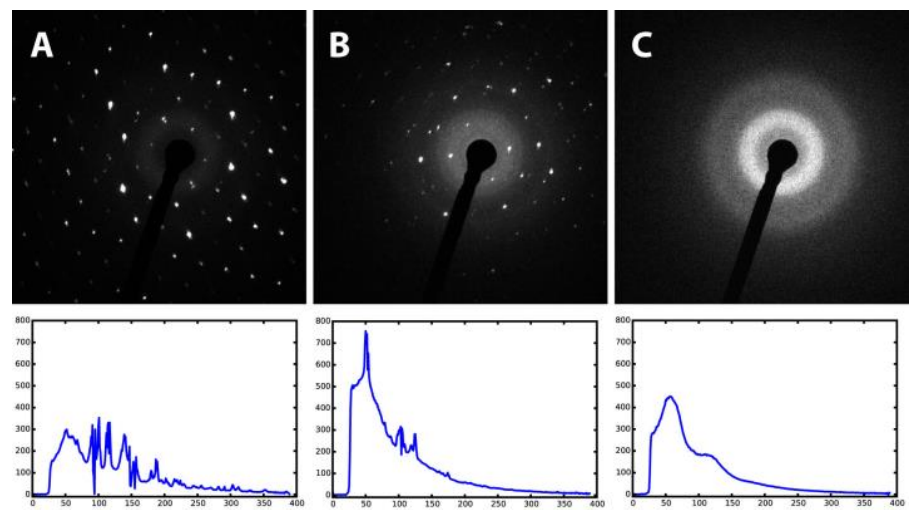

Figure 1. Representative SAED images from (A) fully crystalline material, (B) mixed crystalline and amorphous material, and (C) fully amorphous material. The radial intensity profiles of each pattern is shown beneath.

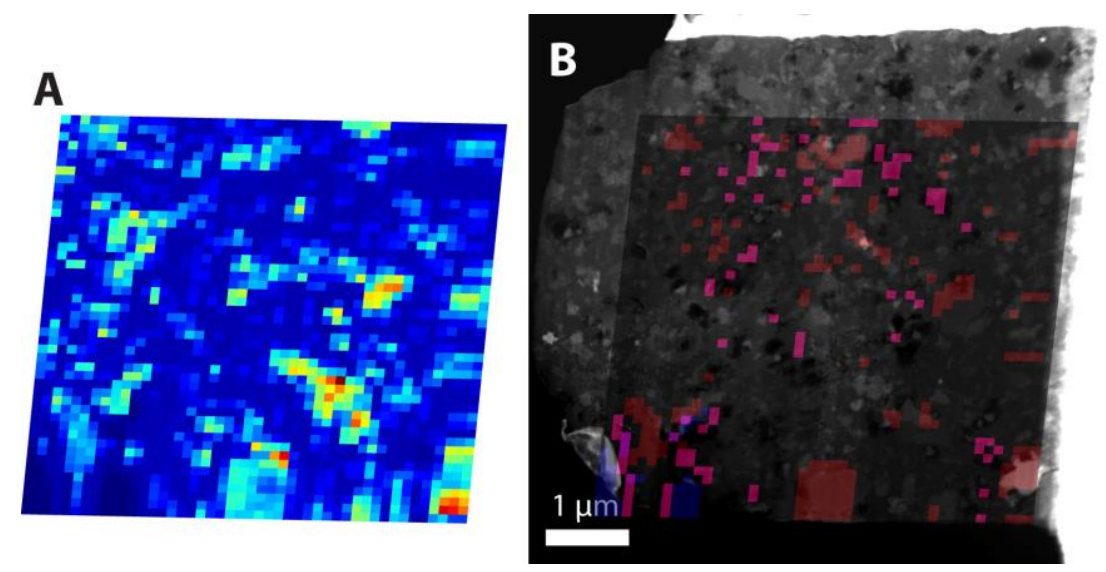

Figure 2. (A) map of diffraction spot intensity across the FIB lamella. (B) HAADF image of the FIB lamella from meteorite ALH77307 with an overlay of the pure amorphous (pink) and isolated crystalline (red) grain maps. 